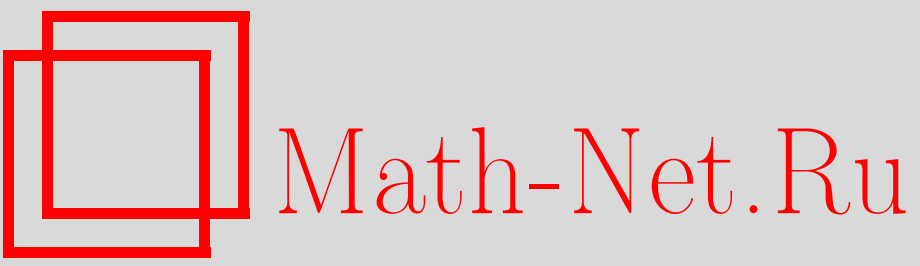

О. А. Олейник, Т. А. Шапошникова, Об усреднении эллиптических задач в перфорированных областях с непериодической структурой, УМН, 1997, том 52, выпуск $6,179-180$

DOI: https://doi.org/10.4213/rm911

Использование Общероссийского математического портала Math-Net.Ru подразумевает, что вы прочитали и согласны с пользовательским соглашением

http://www. mathnet.ru/rus/agreement

Параметры загрузки:

IP: 54.89 .56 .158

26 апреля 2023 г., 08:23:50 


\title{
ОБ УСРЕДНЕНИИ ЭЛЛИПТИЧЕСКИХ \\ ЗАДАЧ В ПЕРФОРИРОВАННЫХ ОБЛАСТЯХ \\ С НЕПЕРИОДИЧЕСКОЙ СТРУКТУРОЙ
}

\author{
О. А. ОЛЕЙНИк, Т. А. ШАПОШНИКОВА
}

1. Здесь мы рассмотрим вопрос об усреднении решений уравнения Пуассона в перфорированной области с непериодической структурой и условиями Неймана или условиями смешанного типа на границе полостей. С лучай периодической структуры области рассмотрен во многих работах и монограффиях (см. [1], [2] и представленную там библиографию).

2. Пусть $\Omega$-гладкая ограниченная область в $\mathbb{R}^{n}$ с границей $\partial \Omega$. Пусть область $G_{\varepsilon}^{j}$ принадлежит $\Omega, j=1, \ldots, N(\varepsilon), \varepsilon$ - мальй параметр, $G_{\varepsilon}^{j} \cap G_{\varepsilon}^{i}=\varnothing$ при $i \neq j, G_{\varepsilon}^{j}$ имеет кусочно-гладкую границу $\partial G_{\varepsilon}^{j}$. Обозначим $|G|$ меру Лебега множества $G$. Положим

$$
G_{\varepsilon}=\sum_{j=1}^{N(\varepsilon)}, \quad \Omega_{\varepsilon}=\Omega \backslash \overline{G_{\varepsilon}}, \quad S_{\varepsilon}^{*}=\partial G_{\varepsilon}, \quad S_{\varepsilon}=S_{\varepsilon}^{*} \cap \Omega, \quad \Gamma_{\varepsilon}=\partial \Omega_{\varepsilon} \backslash S_{\varepsilon}, \quad M_{\varepsilon}=\partial \Omega \cap \overline{G_{\varepsilon}} .
$$

3. Условие Неймана на границе полостей. Рассмотрим в $\Omega_{\varepsilon}$ краевую задачу

$$
-\Delta u_{\varepsilon}=f \text { в } \Omega_{\varepsilon}, \quad \frac{\partial u_{\varepsilon}}{\partial \nu}=0 \text { на } S_{\varepsilon}, \quad u_{\varepsilon}=0 \text { на } \Gamma_{\varepsilon},
$$

где $\nu$ - внешняя единичная нормаль к $S_{\varepsilon}, f$ - гладкая функция в $\bar{\Omega}$.

Под обобщенным решением задачи (1) мы понимаем функцию $u_{\varepsilon}$ из пространства $H_{1}\left(\Omega_{\varepsilon}, \Gamma_{\varepsilon}\right)$. Здесь через $H_{1}(\omega, \gamma)$ мы обозначили пополнение бесконечно дифференцируемых в $\bar{\omega}$ функций, равных нулю в окрестности $\gamma$, по норме

$$
\|u\|_{H_{1}(\omega, \gamma)}^{2}=\int_{\omega}\left(|\nabla u|^{2}+u^{2}\right) d x, \quad \nabla u \equiv\left(\frac{\partial u}{\partial x_{1}}, \ldots, \frac{\partial u}{\partial x_{n}}\right) .
$$

Будем предполагать, что любую функцию $u \in H_{1}\left(\Omega_{\varepsilon}, \Gamma_{\varepsilon}\right)$ можно продолжить на $\Omega$ как функцию $\widetilde{u} \in H_{1}\left(\Omega, \Gamma_{\varepsilon}\right)$ так, что выполняются неравенства

$$
\|\widetilde{u}\|_{H_{1}(\Omega)} \leqslant K_{1}\|u\|_{H_{1}(\Omega)}, \quad\|\nabla \widetilde{u}\|_{L_{2}(\Omega)} \leqslant K_{2}\|\nabla u\|_{L_{2}\left(\Omega_{\varepsilon}\right)}
$$

где постоянные $K_{j}$ здесь и далее не зависят от $\varepsilon$. Будем также предполагать, что для $u \in H_{1}\left(\Omega_{\varepsilon}, \Gamma_{\varepsilon}\right)$ вьполнено неравенство Фридрихса

$$
\|u\|_{L_{2}(\Omega)} \leqslant K_{3}\|\nabla u\|_{L_{2}\left(\Omega_{\varepsilon}, \Gamma_{\varepsilon}\right)},
$$

Случаи, когда эти предположения вьполнены, указаны в [1]. Пусть $v_{0}$ - решение задачи

$$
-\Delta v_{0}=f \text { в } \Omega, \quad v_{0}=0 \text { на } \partial \Omega .
$$

Теорема 1. Пусть $u_{\varepsilon}-$ решение задачи (1), $v_{0}$ - решение задачи (4). Тогда

$$
\begin{array}{lll}
\left\|u_{\varepsilon}-v_{0}\right\|_{H_{1}\left(\Omega_{\varepsilon}\right)} \leqslant K_{4}\left|G_{\varepsilon}\right|^{1 / 2}, & \text { ecлu } & S_{\varepsilon}^{*} \cap \partial \Omega=\varnothing, \\
\left\|u_{\varepsilon}-v_{0}\right\|_{H_{1}\left(\Omega_{\varepsilon}\right)} \leqslant K_{5}\left(\left|G_{\varepsilon}\right|^{1 / 2}+\left|M_{\varepsilon}\right|^{1 / 2}\right), & \text { ecлu } & S_{\varepsilon} \cap \partial \Omega \neq \varnothing .
\end{array}
$$


ПримеРы. Пусть $G_{\varepsilon}^{j}$ имеет диаметр $a_{\varepsilon}^{j}$ и $N(\varepsilon) \leqslant d_{s} \varepsilon^{-s}, 0 \leqslant s \leqslant n, d_{s}=$ const $>0$. Этот случай включает области, перфорированные вдоль многообразий $M^{s} \subset \Omega$ размерности $s, s<n$, либо $G_{\varepsilon}^{j}$ могут быть расположены произвольным образом внутри $\Omega, s=n$. Тогда

$$
\begin{aligned}
& \left\|u_{\varepsilon}-v_{0}\right\|_{H_{1}\left(\Omega_{\varepsilon}\right)} \leqslant K_{6} \max _{j}\left|a_{\varepsilon}^{j}\right|^{n} \varepsilon^{-s}, \text { если } S_{\varepsilon}^{*} \cap \partial \Omega=\varnothing, \\
& \left\|u_{\varepsilon}-v_{0}\right\|_{H_{1}\left(\Omega_{\varepsilon}\right)} \leqslant K_{7}\left(\max _{j}\left|a_{\varepsilon}^{j}\right|^{n-1} \varepsilon^{-k}+\max _{j}\left|a_{\varepsilon}^{j}\right|^{n} \varepsilon^{-s}\right),
\end{aligned}
$$

если $S_{\varepsilon}^{*} \cap \partial \Omega \neq \varnothing$ и число полостей $G_{\varepsilon}^{j}$ таких, что $\partial \Omega \cap \partial G_{\varepsilon}^{j}$, не превосходит $d_{k} \varepsilon^{-k}$, $N(\varepsilon) \leqslant d_{s} \varepsilon^{-s}$.

\section{4. Смешанные граничные условия. Рассмотрим задачу}

$$
-\Delta u_{\varepsilon}=f \quad \text { в } \Omega_{\varepsilon}, \quad \frac{\partial u_{\varepsilon}}{\partial \nu}+\beta(x) u_{\varepsilon}=0 \text { на } S_{\varepsilon}, \quad u_{\varepsilon}=0 \text { на } \Gamma_{\varepsilon} .
$$

Теорема 2. Пусть $\beta(x) \geqslant \beta_{0}>0, u_{\varepsilon}$ - обобщенное решение задачи (5), $v_{0}$ - решение задачи (4). Тогда

$$
\left\|u_{\varepsilon}-v_{0}\right\|_{H_{1}\left(\Omega_{\varepsilon}\right)}^{2} \leqslant K_{8}\left|S_{\varepsilon}\right| .
$$

ПримеРы. Пусть $\left.\mid \partial G_{\varepsilon}^{j}\right) \mid \leqslant K_{9}\left(a_{\varepsilon}^{j}\right)^{n-1}, a_{\varepsilon}^{j}-$ диаметр области $G_{\varepsilon}^{j}$ и пусть $N(\varepsilon) \leqslant d_{s} \varepsilon^{-s}$. Тогда справедлива оценка

$$
\left\|u_{\varepsilon}-v_{0}\right\|_{H_{1}\left(\Omega_{\varepsilon}\right)}^{2} \leqslant K_{10}\left(\max _{j} a_{\varepsilon}^{j}\right)^{n-1} \varepsilon^{-s} .
$$

Задача усреднения с условиями Дирихле на границе полостей и усреднение некоторых задач смешанного типа мы рассмотрим в следующей статье.

\section{СПИСОК ЛИТЕРАТУРЫ}

[1] Жииков В. В., Козлов С. М., Олейник О. А. Усреднение дифференциальных операторов. М.: Физматлит, 1993. [2] Bensoussan A., Lions J. L., Papanicolau G. Asymptotic Analysis for Periodic Structure. Amsterdam: North Holland, 1978.

Московский государственный

Принято редколлегией университет им. М. В. Ломоносова 